\title{
Number of Transition Frequencies of a System Containing an Arbitrary Number of Gas Bubbles
}

\author{
Masato Ida \\ Satellite Venture Business Laboratory, Gunma University, 1-5-1 Tenjin-cho, Kiryu-shi, Gunma 376-8515, Japan
}

\begin{abstract}
"Transition frequencies" of a system containing an arbitrary number of bubbles levitated in a liquid are discussed. Using a linear coupled-oscillator model, it is shown theoretically that when the system contains $N$ bubbles of different sizes, each bubble has $2 N-1$ (or less) transition frequencies which make the phase difference between an external sound and a bubble's pulsation $\pi / 2$. Furthermore, we discuss a discrepancy appearing between the present result regarding the transition frequencies and existing ones for the resonance frequencies in a two-bubble case, and show that the transition frequency, defined as above, and the resonance frequency have a different physical meaning when $N \geq 2$, while they are consistent for $N=1$.
\end{abstract}

Multibubble dynamics in an acoustic field have been studied in a variety of fields [1-13], and the mutual radiative interaction of bubbles is known to change their acoustic properties [1] 6, 8, 9, 12]. Shima [1] derived a theoretical formula for the resonance frequencies of two mutually interacting gas bubbles levitated in an inviscid liquid, and showed that a bubble interacting with a neighboring bubble has two resonance frequencies, which reveal an upward or downward shift as the bubbles approach each other. Zabolotskaya [2] showed theoretically that variations in the resonance frequencies may cause changes of phase difference between bubbles, and may, in turn, sometimes give rise to reversal of the sign of the secondary Bjerknes force, which is an interaction force acting between pulsating bubbles [10]. In the studies described in refs. 3 and 4, Doinikov and Zavtrak produced results that were almost equivalent to those of Zabolotskaya by employing a mathematical model in which the multiple scattering between bubbles is described using a Legendre expansion function. Their results are considered to explain the "bubble grape", which is a stable structure formed by bubbles in an acoustic field [ 4 . Feuillade [5], using a self-consistent model, showed that when two bubbles are pulsating in antiphase and radiation damping is dominant rather than thermal or viscous damping, the bubbles may show a superresonant response [6] because of the reduction in the effective damping. Ye and Alvarez [7, using a self-consistent model, carried out numerical experiments on acoustic wave propagation in a liquid containing randomly distributed identical bubbles, and showed that the localization of acoustic waves can take place in a range of frequencies. It was considered that the multiple scattering of sound due to the bubbles has a strong influence on this phenomenon. Mettin et al. [8] and Doinikov [9] examined some influences of the interaction of two bubbles in a strong acoustic field on the radiation forces (the secondary [10] and primary 114,15 Bjerknes forces) acting on the bubbles; these studies are related to multibubble sonoluminescence [16,17].

The aim of this paper is to provide preliminary but significant discussions regarding the "transition frequency" of a system containing an arbitrary number of bubbles having different radii, by employing a simple theoretical model. In the present study, the transition frequencies of a bubble in the system are defined as the frequencies of an external sound for which the phase difference between the sound and the bubble's pulsation becomes $\pi / 2$ [12]. This definition is based on the well-known phenomenon of a single bubble case in which the phase difference between an external sound and the bubble pulsation becomes $\pi / 2$ at its resonance frequency (more correctly, at its natural frequency) [13,18]. This study has two main purposes: (1) To determine how many transition frequencies a bubble in the system has. As has been reported, it can be considered that the phase shifts of the bubble pulsations due to the radiation coupling give rise to a large variety of phenomena that cannot be predicted by a single-bubble model (see, e.g., refs. 1-9). However, there exist only a few studies on the transition frequencies defined as mentioned above. Almost all of the previous works (e.g., refs. 1-5) concern the resonance frequencies. Very recently, Ida 12], using a coupled oscillator model, showed theoretically that a bubble interacting with a neighboring bubble has three transition frequencies. In the present paper, by extending this approach, we derive the number of transition frequencies of a bubble interacting with an arbitrary number of bubbles. (2) To elucidate a difference between the transition frequency and the resonance frequency. As suggested already, the transition frequency and the resonance frequency have different physical meanings when the number of bubbles, $N$, is larger than 1. As briefly reviewed above, Shima [1] and Zabolotskaya [2] predicted the existence of two resonance frequencies per bubble when $N=2$. This number of resonance frequencies is not equal to that of the transition frequencies given by Ida 12]. In the present paper, we show that in general, the numbers of those frequencies are different. This result shows that when $N \geq 2$, the reversal of the phase of the bubble pulsation (e.g., from in-phase to out-of-phase with an external sound) may occur not only at the resonance but also at other frequencies, unlike the case of $N=1$.

At first, we derive a system of linear differential equations that describes the pulsation of coupled bubbles and that is almost equivalent to those used in refs. 1, 2, 5, 12 and 19. Let us assume that $N$ bubbles are levitated in a 
viscous liquid and the equilibrium radius of each bubble (assumed to be constant [20]) is $R_{i 0}(i=1,2, \cdots, N)$. When the amplitude of the external sound is weak, the bubble pulsation can be described by a linear secondorder differential equation [21,22,13,

$$
\ddot{e}_{i}+\omega_{i 0}^{2} e_{i}+\delta_{i} \dot{e}_{i}=-\frac{1}{\rho R_{i 0}} p_{\mathrm{d} i}
$$

where it is assumed that the sphericity of the bubbles is maintained and the time-dependent bubble radii $R_{i}(t)$ can be represented by $R_{i}(t)=R_{i 0}+e_{i}(t)\left(\left|e_{i}\right| \ll R_{i 0}\right)$, and $\omega_{i 0}\left(=\sqrt{\left[3 \kappa P_{0}+(3 \kappa-1) 2 \sigma / R_{i 0}\right] / \rho R_{i 0}^{2}}\right)$ is the resonance (angular) frequency, $\delta_{i}$ is the damping factor [23], $p_{\mathrm{d} i}$ is the driving pressure acting on bubble $i, \rho$ is the density of the surrounding liquid, $\kappa$ is the polytropic exponent of the gas inside the bubbles, $P_{0}$ is the equilibrium pressure, $\sigma$ is the surface-tension coefficient, and the over dots denote the derivation with respect to time. When more than one bubble exists, the driving pressure $p_{\mathrm{d} i}$ is given by the sum of the pressures of the external sound, $p_{e x}$, and the scattered sound waves due to other bubbles, $p_{\mathrm{s} i j}$, as 24

$$
p_{\mathrm{d} i}=p_{\mathrm{ex}}+\sum_{j=1, j \neq i}^{N} p_{\mathrm{s} i j} \approx p_{\mathrm{ex}}+\sum_{j=1, j \neq i}^{N} \frac{\rho R_{j 0}^{2}}{D_{i j}} \ddot{e}_{j},
$$

where the surrounding liquid is assumed to be incompressible, and $D_{i j}$ is the distance between the centers of bubbles $i$ and $j$. By substituting eq. (2) into eq. (1), one obtains

$$
\ddot{e}_{i}+\omega_{i 0}^{2} e_{i}+\delta_{i} \dot{e}_{i}=-\frac{p_{\mathrm{ex}}}{\rho R_{i 0}}-\frac{1}{R_{i 0}} \sum_{j=1, j \neq i}^{N} \frac{R_{j 0}^{2}}{D_{i j}} \ddot{e}_{j} .
$$

This kind of system of differential equations is called a coupled-oscillator model or a self-consistent model. Now we assume that $p_{\mathrm{ex}}=P \exp (\mathrm{i} \omega t)$ and $e_{i}=A_{i} \exp (\mathrm{i} \omega t)$, where $P$ is a real, positive constant and $A_{i}$ denotes complex amplitudes. Those assumptions reduce eq. (3) to

$$
R_{i 0}\left[\left(\omega^{2}-\omega_{i 0}^{2}\right)-\mathrm{i} \omega \delta_{i}\right] A_{i}+\sum_{j=1, j \neq i}^{N} \frac{R_{j 0}^{2}}{D_{i j}} \omega^{2} A_{j}=\frac{P}{\rho} .
$$

This system of equations can be written in a matrix form,

$$
\mathbf{M A}=\mathbf{B}
$$

where $\mathbf{M}$ is an $N \times N$ matrix whose elements, $m_{i j}$, are defined as

$$
m_{i j}= \begin{cases}R_{i 0}\left[\left(\omega^{2}-\omega_{i 0}^{2}\right)-\mathrm{i} \omega \delta_{i}\right], & \text { for } i=j, \\ \frac{R_{j 0}^{2}}{D_{i j}} \omega^{2}, & \text { otherwise }\end{cases}
$$

$\mathbf{A}=\left(A_{1}, A_{2}, \cdots, A_{N}\right)^{T}$ and $\mathbf{B}=(P / \rho)(1,1, \cdots, 1)^{T}$.

Let us analyze the matrix equation. The solution of eq. (5) is expressed as

$$
\mathbf{A}=\mathbf{M}^{-1} \mathbf{B}
$$

As is well known, $\mathbf{M}^{-1}$ can be written in the form of

$$
\mathbf{M}^{-1}=\frac{\mathbf{C}}{|\mathbf{M}|}
$$

where $|\mathbf{M}|$ and $\mathbf{C}$ are the determinant and the cofactor matrix, respectively, of $\mathbf{M}$. From the definitions of the determinant and the cofactor of a matrix, one determines that

$$
\begin{gathered}
\operatorname{deg}(|\mathbf{M}(\omega)|)=2 N, \\
\operatorname{deg}(\mathbf{C}(\omega))=2(N-1) .
\end{gathered}
$$

Here we should note that $\operatorname{deg}\left(m_{i j}(\omega)\right)=2$. Substitution of eq. (8) into eq. (7) yields

$$
\mathbf{A}=\frac{1}{|\mathbf{M}|} \mathbf{C} \mathbf{B}
$$

As defined previously, the transition frequencies of bubble $i$ are derived by an equation of

$$
\operatorname{Re}\left(A_{i}\right)=0
$$

Assuming that

$$
\begin{aligned}
& |\mathbf{M}|=a+\mathrm{i} b, \\
& g_{i}=c_{i}+\mathrm{i} d_{i},
\end{aligned}
$$

where $a, b, c_{i}$, and $d_{i}$ are real values and $g_{i}$ is an element of $\mathbf{C ~ B}$, yields

$$
A_{i}=\frac{c_{i}+\mathrm{i} d_{i}}{a+\mathrm{i} b}=\frac{a c_{i}+b d_{i}+\mathrm{i}\left(a d_{i}-b c_{i}\right)}{a^{2}+b^{2}}
$$

and

$$
\operatorname{Re}\left(A_{i}\right)=\frac{a c_{i}+b d_{i}}{a^{2}+b^{2}} .
$$

Using this equation and assuming that $|\mathbf{M}| \neq 0$, i.e., no case exists where both $a=0$ and $b=0$ are true (this assumption is physically valid because this guarantees the absence of a singular solution), eq. (11) is reduced to

$$
a c_{i}+b d_{i}=0
$$

Now we examine how many roots this equation has. From eqs. (9) and (10) and definitions (12) and (13), we know that $\operatorname{deg}\left(a c_{i}+b d_{i}\right)=2 N+2(N-1)=4 N-2$. Furthermore, using definition (6), we can prove that eq. (15) contains terms of only even orders with respect to $\omega$ as follows: the real parts of $m_{i j}$ are of the second order and the imaginary ones are of the first order with respect to $\omega$; thus, only terms of even orders remain in the real part of $A_{i}$, because an even-order component given by the imaginary parts of $m_{i j}$, e.g., $\mathrm{i} \omega \delta_{1} \times \mathrm{i} \omega \delta_{2}=-\delta_{1} \delta_{2} \omega^{2}$, becomes a real value and an odd-order component, e.g., 
$\mathrm{i} \omega \delta_{1} \times \mathrm{i} \omega \delta_{2} \times \mathrm{i} \omega \delta_{3}=-\mathrm{i} \delta_{1} \delta_{2} \delta_{3} \omega^{3}$, becomes an imaginary value. As a result, eq. (15) can be represented by

$$
F(X) \equiv a c_{i}+b d_{i}=0,
$$

and one knows that

$$
\operatorname{deg}(F(X))=2 N-1
$$

with $X=\omega^{2}$. These equations predict that a bubble has $2 N-1$ (or less) transition frequencies. (Note that only a positive root is physical.) When $N=2$, for example, $2 N-1=3$; this result is in agreement with that given by Ida 12 .

To confirm the above result, we show here a numerical result for $N=3$. The parameters used are $R_{10}=1 \mu \mathrm{m}$, $R_{20}=1.5 \mu \mathrm{m}, R_{30}=2.5 \mu \mathrm{m}, \rho=1000 \mathrm{~kg} / \mathrm{m}^{3}, \kappa=1.4$, $P_{0}=1 \mathrm{~atm}$, and $\sigma=0.0728 \mathrm{~N} / \mathrm{m}$. The damping factor is set to $\delta_{i}=4 \mu / \rho R_{i 0}^{2}$, where $\mu\left(=1.137 \times 10^{-3} \mathrm{~kg} /(\mathrm{m}\right.$ $\mathrm{s})$ ) is the viscosity of water at room temperature (i.e., the viscous damping is adapted, which is dominant for small bubbles used, e.g., in medical applications [11] and in the experiments on sonoluminescence 16.13), and $D_{i j}$ is determined by $D_{i j}=s\left(R_{i 0}+R_{j 0}\right)$. For $s=2.0$, we, as expected, obtain five $(=2 \times 3-1)$ transition frequencies of bubble $1, \omega_{1}=(1.054,0.723,0.635,0.411,0.328) \times \omega_{10}$, while for $s=10.0$, only one, $\omega_{1}=1.002 \omega_{10}$, is found. Such a dependency of the number on the distance between bubbles can also be found in a two-bubble case 12. Detailed discussion regarding three- or more-bubble cases will be given in a future paper.

We perform here a comparative study between the present theory and previous ones in order to clarify a difference between the transition frequency and the resonance frequency. Shima [1] and Zabolotskaya [2] derived the same formula for estimating the resonance frequencies in the case of $N=2$, expressed as

$$
\left(X-\omega_{10}^{2}\right)\left(X-\omega_{20}^{2}\right)-\frac{R_{10} R_{20}}{D_{21}^{2}} X^{2}=0 .
$$

In their mathematical models, the sphericity of bubbles and incompressibility of the surrounding liquid were assumed as in the present one, but the damping factor was neglected. This formula predicts two resonance frequencies; apparently, this result seems to contradict the result given by Ida, which predicts three transition frequencies 12. We next discuss the origin of this discrepancy.

Generally, when no damping factor exists $\left(\delta_{i}=0\right.$, $\left.\operatorname{Im}\left(m_{i j}\right)=0\right)$, the resonance frequencies are determined by

$$
|\mathbf{M}|=0
$$

in the case of this study. This condition gives rise to the well-known singularity in the bubble pulsation at the resonance frequencies (infinite amplitudes and discontinuous phase reversals 25,14. This formula is reduced to the well-known one for a single-bubble case, $\omega^{2}-\omega_{10}^{2}=0$, and to eq. (18) for a double-bubble case. On the other hand, in the present study, the transition frequencies of bubble $i$ in the case of $\delta_{i}=0$ are derived by the following independent equations:

$$
\operatorname{Re}\left(A_{i}\right)=A_{i}=\frac{g_{i}}{|\mathbf{M}|}=0
$$

and

$$
\operatorname{Sign}\left(\lim _{\Delta \rightarrow-0} A_{i}(\omega+\Delta)\right) \neq \operatorname{Sign}\left(\lim _{\Delta \rightarrow+0} A_{i}(\omega+\Delta)\right)
$$

where

$$
\operatorname{Sign}(f)= \begin{cases}1 & \text { for } f>0 \\ -1 & \text { for } f<0\end{cases}
$$

Equation (21) means that the phase of bubble $i$ is reversed at $\omega$ satisfying this equation. (In the limit of $\delta_{i} \rightarrow 0$, the condition that the phase difference is $\pi / 2$ converges to eq. (21) at the resonances because of the singularity mentioned above. Namely, at the resonances those conditions are essentially equivalent.) Equations (20) and (21), respectively, may be reduced to $g_{i}=0$ and $|\mathbf{M}|=0$, or

$$
|\mathbf{M}| g_{i}=0 .
$$

Furthermore, using the definitions of eqs. (12) and (13), eq. (22) can be reduced to

$$
a c_{i}=0 \text {. }
$$

This equation is in agreement with eq. (15) when $b=0$ and $d_{i}=0$ (these conditions are naturally satisfied because of $\left.\operatorname{Im}\left(m_{i j}\right)=0\right)$. The degree of eq. (19) with respect to $X$ is $N$ as discussed above, while that of eq. (22) is $N+(N-1)=2 N-1$. When $N>1$, these two degrees are not equal to one another. This result reveals that (1) "resonance frequency" and "transition frequency" have different physical meanings, (2) the number of the transition frequencies is, in general, larger than that of the resonance frequency, and (3) other conditions under which the phase reversal of bubble pulsation takes place exist in addition to those for the resonance. The transition frequencies given by $|\mathbf{M}|=0$ cause the resonance response of the bubbles, while those given by $g_{i}=0$ do not cause it. (The respective transition frequencies may correspond to the series and the parallel resonance frequencies of an electromagnetic circuit, which give rise to a zero and an infinite impedance, respectively.)

Next, we discuss the case of $D_{i j} \approx \infty$ and $\delta_{i} \approx 0$, i.e., in which the non-diagonal elements of $\mathbf{M}$ and the imaginary parts of its diagonal elements have quite small absolute values. These settings result in

$$
\begin{array}{ll}
a=\prod_{j=1}^{N} R_{j 0}\left(\omega^{2}-\omega_{j 0}^{2}\right)+\varepsilon 1, & b=\varepsilon 2, \\
c_{i}=\frac{P}{\rho} \prod_{j=1, j \neq i}^{N} R_{j 0}\left(\omega^{2}-\omega_{j 0}^{2}\right)+\varepsilon 3, & d_{i}=\varepsilon 4,
\end{array}
$$


where $\varepsilon 1 \sim \varepsilon 4$ are real values whose absolute values are quite small. They reduce eq. (15) to

$$
\left(\prod_{j=1}^{N} R_{j 0}\left(\omega^{2}-\omega_{j 0}^{2}\right)\right)\left(\frac{P}{\rho} \prod_{j=1, j \neq i}^{N} R_{j 0}\left(\omega^{2}-\omega_{j 0}^{2}\right)\right) \approx 0
$$

or

$$
\left(\omega^{2}-\omega_{i 0}^{2}\right) \prod_{j=1, j \neq i}^{N}\left(\omega^{2}-\omega_{j 0}^{2}\right)^{2} \approx 0
$$

This equation shows that for $D_{i j} \rightarrow \infty$, among $2 N-1$ transition frequencies of bubble $i$, only one converges to $\omega_{i 0}$, while two of the remaining ones converge to $\omega_{j 0}$ $(j \neq i)$. This result is consistent with that for $N=2$. 12

Lastly, we briefly discuss a special case. Here we assume that all bubbles are identical $\left(R_{10}=R_{20}=\cdots=\right.$ $\left.R_{N 0}, \delta_{1}=\delta_{2}=\cdots=\delta_{N}\right)$ and, furthermore, that the distances between all pairs of bubbles are uniform. (The latter assumption may be realizable up to $N=4$.) When $N=3$, this setting corresponds to the "mode $A$ " discussed in Sec. III of ref. 5. Based on these assumptions, the elements of matrix $|\mathbf{M}|$ become

$$
\begin{gathered}
m_{11}=m_{22}=\cdots=m_{N N}=R_{10}\left[\left(\omega^{2}-\omega_{10}^{2}\right)-\mathrm{i} \omega \delta_{1}\right] \\
m_{12}=m_{21}=m_{13}=\cdots m_{N N-1}=\frac{R_{10}^{2}}{D_{12}} \omega^{2}
\end{gathered}
$$

Namely, both the diagonal and the non-diagonal elements become uniform. As a result, one obtains $A_{1}=A_{2}=$ $\cdots=A_{N}$ and

$$
A_{1}=\frac{1}{m_{11}+(N-1) m_{12}} \cdot \frac{P}{\rho} .
$$

Only one transition frequency (corresponding to the resonance frequency) is given by this formula, although $2 N-1$ ones are predicted in general. This result points out that the numbers of both the resonance and the transition frequencies are reduced under certain conditions and that such an excessive idealization prevents the accurate understanding of multibubble dynamics in an acoustic field.

In summary, we have studied the transition frequencies of a system containing an arbitrary number $(N)$ of bubbles of different sizes by employing a linear coupledoscillator model. In the present study, the frequency of an external sound in the case where the phase difference between the external sound and a bubble's pulsation becomes $\pi / 2$ was called the transition frequency. The present theory predicts that a bubble in the system has $2 N-1$ transition frequencies, while it has $N$ resonance frequencies; namely, the well-known theory for a one-bubble case in which the phase reversal of a bubble's pulsation takes place around its resonance frequency is not absolutely true in a two- or more-bubble case. (The physical meaning of the existence of an odd number of transition frequencies may be explained as follows: a bubble, even if interacting with neighboring bubbles, pulsates in-phase or out-of-phase with an external sound if the driving frequency is much lower or much higher, respectively, than the resonance frequencies of the bubbles; to interpolate those two extreme states consistently, an odd number of the phase reversals is needed.) This result is important especially for subjects in which the phase change of bubbles plays an important role, such as those studied in refs. 2-4, 8 and 9 and investigated using only the resonance frequencies. (In ref. 26, the author already applied the theory for the transition frequency to the investigation of the reversal of the sign of the secondary Bjerknes force, and found that the theory gives an alternative interpretation of the phenomenon, which may be more accurate than the previous ones given in refs. 2-4.) We expect that more detailed mathematical discussions regarding the matrix equation will supply a still richer understanding of the physics of multibubble dynamics in an acoustic field and acoustic wave propagation in bubbly liquid.

[1] A. Shima: Trans. ASME, J. Basic Eng. 93 (1971) 426.

[2] E. A. Zabolotskaya: Sov. Phys. Acoust. 30 (1984) 365.

[3] A. A. Doinikov and S. T. Zavtrak: Phys. Fluids 7 (1995) 1923.

[4] A. A. Doinikov and S. T. Zavtrak: J. Acoust. Soc. Am. 99 (1996) 3849.

[5] C. Feuillade: J. Acoust. Soc. Am. 98 (1995) 1178.

[6] I. Tolstoy: J. Acoust. Soc. Am. 80 (1986) 282.

[7] Z. Ye and A. Alvarez: Phys. Rev. Lett. 80 (1998) 3503.

[8] R. Mettin, I. Akhatov, U. Parlitz, C. D. Ohl and W. Lauterborn: Phys. Rev. E 56 (1997) 2924.

[9] A. A. Doinikov: Phys. Rev. E 62 (2000) 7516.

[10] L. A. Crum: J. Acoust. Soc. Am. 57 (1975) 1363.

[11] P. A. Dayton, K. E. Morgan, A. L. Klibanov, G. Brandenburger, K. R. Nightingale and K. W. Ferrara: IEEE Trans. Ultrason. Ferroelect. \& Freq. Control 44 (1997) 1264.

[12] M. Ida (accepted for publication in Phys. Lett. A); the linear theory given in this paper for deriving the transition frequencies is also shown in e-Print, physics/0108067. (The transition frequencies are called "eigenfrequencies" in this report.)

[13] W. Lauterborn, T. Kurz, R. Mettin and C. D. Ohl: Adv. Chem. Phys. 110 (1999) 295.

[14] A. I. Eller: J. Acoust. Soc. Am. 43 (1968) 170.

[15] L. A. Crum and A. I. Eller: J. Acoust. Soc. Am. 48 (1969) 181.

[16] L. A. Crum: Phys. Today 47 No. 9 (1994) 22.

[17] K.Yasui: Phys. Rev. Lett. 83 (1999) 4297.

[18] T. G. Leighton: The Acoustic Bubble (Academic Press, London, 1994), p. 293. 
[19] C. Feuillade: J. Acoust. Soc. Am. 109 (2001) 2606.

[20] In theoretical and numerical investigations of bubble dynamics in an acoustic field, evaporation and condensation occurring inside bubbles, which result in the change in the equilibrium radii, have been neglected except for some special cases, since those processes take place very slowly compared to the period of the external sound of practical interest. 21]. (For readers interested in the modeling of those processes, we recommend I. Akhatov, O. Lindau, A. Topolnikov, R. Mettin, N. Vakhitova and W. Lauterborn: Phys. Fluids 13 (2001) 2805 and references therein.)

[21] A. Prosperetti: Ultrasonics 22 (1984) 69.

[22] T. G. Leighton: The Acoustic Bubble (Academic Press, London, 1994), p. 291.

[23] Generally, $\delta_{i}$ is determined by the sum of the viscous, acoustic and thermal damping coefficients, the second and the last ones of which depend on the driving frequency 21.5. The present paper, however, treats $\delta_{i}$ as being independent of the frequency, to simplify the discussion. The numerical results regarding two-bubble cases 261 reveal that even if the acoustic and thermal damping are taken into account, the maximum number of the transition frequencies does not change.

[24] The amplitude of the scattered wave can be given, e.g., by integrating the momentum equation for linear sound waves, $\partial p / \partial r=-\rho \partial u / \partial t$, coupled with the divergencefree condition, $\partial\left(r^{2} u\right) / \partial r=0$, where $r$ is the radial coordinate measured from the center of a bubble and $u$ is the velocity along $r$.

[25] M. Minnaert: Phil. Mag. 16 (1933) 235.

[26] M. Ida (submitted); e-Print, physics/0109005. 